\title{
Commentary \\ Recently published papers: Heavyweight problems in the intensive care unit?
}

\author{
Lui G Forni ${ }^{1,2}$
}

1Department of Critical Care, Worthing General Hospital, Lyndhurst Road, Worthing BN11 2DH, UK

${ }^{2}$ Brighton \& Sussex Medical Schools, University of Sussex, Brighton BN1 9PX, UK

Corresponding author: Lui G Forni, Lui.Forni@wash.nhs.uk

Published: 10 December 2008

This article is online at http://ccforum.com/content/12/6/197

Critical Care 2008, 12:197 (doi:10.1186/cc7138)

(c) 2008 BioMed Central Ltd

\begin{abstract}
The management of the obese patient in intensive care is fraught with difficulty, or so conventional wisdom would have us believe. Two recent studies add to the growing evidence that the obese patient is not significantly disadvantaged in terms of outcome following intensive care admission. Another study highlights the increasing burden of end-stage renal disease on intensive care unit resources with some interesting results. And there is more on citrate, the new anticoagulant!
\end{abstract}

Thou seest I have more flesh than another man, and therefore more frailty.

(William Shakespeare)

The imbalance between the haves and the have nots is no more striking than when one considers nutrition. Obesity is an increasing problem in most of the western world with approaching 300 million people being classified as obese, whereas starvation affects almost one-half of the global population. Indeed, the obesity epidemic is highlighted here in the United Kingdom, where over the period between 1980 and 1997 the obesity rates in adults trebled [1]. As a consequence we are often faced with the management of such patients in our intensive care units (ICUs) and their inherent problems. Obese patients are viewed as more complex to manage for a variety of reasons: they are difficult to intubate, difficult to ventilate, difficult to wean, difficult to move - the list goes on. And on. But are these generalisations justified in the majority of cases? Two studies reported recently in Intensive Care Medicine add more weight to the arguments against such views $[2,3]$.

In the study by Sakr and colleagues, the European observational Sepsis Occurrence in Acutely III Patients study database was interrogated to examine the effect of body mass index (BMI) on morbidity and mortality outcomes [2]. Patients were stratified as underweight (BMI <18.5), normal weight (BMI 18.5 to 24.9), overweight (BMl 25 to 29.9), obese (BMI 30 to 39.9 ) and very obese (BMI $\geq 40$ ). BMI data were available from $91 \%$ of the cohort, with $53.9 \%$ being classed as overweight or greater and $2.8 \%$ classed as very obese. The results make interesting reading. There was no association between increased mortality and obesity. There was, however, an increase in morbidity with a trend towards longer length of stay, both in the ICU and in the hospital, between the normal weight groups and the very obese patients. Similarly, those patients with higher BMI showed an increase in ICU-acquired infections. The authors do point out the limitations of this study, not least the inconsistencies in measurement of height and weight (a simple yet seemingly impossible task to do well in the ICU) and the paucity of detailed data relating to mechanical ventilation parameters, including the use of tracheotomies [2].

Frat and colleagues have examined the effect of obesity on mechanically ventilated patients prospectively, which makes their study almost unique [3]. Patients were classified into two groups, severely obese (BMI $\geq 35$ ) or normal (BMI <30). Again, no differences in mortality were observed - although intubation was more difficult and stridor on extubation was a more common complication in the severly obese patients. So where does this leave us? As doctors we are not immune from society's prejudice against obese individuals, and some intensivists may still share Shakespeare's view - but in the light of these recent studies, perhaps it is time for a rethink. Indeed, when one considers the BMI and ICU outcome, it is the malnourished patients $(\mathrm{BMl}<18.5)$ who fare worse, not the obese patients - a thought-provoking point made in the accompanying editorial $[4,5]$.

$\mathrm{BMI}=$ body mass index; $\mathrm{ICU}=$ intensive care unit. 
One conclusion from these studies is clear: obese patients do consume considerable resources in common with another group of patients - those requiring end-stage renal support. In keeping with, and probably intimately linked to, obesity, the need for end-stage renal support is increasing - with some sources predicting a doubling in incidence over the next decade [6]. Unsurprisingly, ICU admissions of patients with end-stage renal disease are increasing, and a recent UK study revealed that $1.3 \%$ of all patients admitted to the ICU were on end-stage renal support [7].

A recent study from the Swedish Intensive Care Nephrology Group (SWING) investigators examined both short-term and long-term outcomes of patients admitted to the ICU already on end-stage renal support in Sweden [8]. Interestingly the study showed that the 90-day mortality for patients with endstage renal disease admitted to the ICU was $42 \%$, compared with $50 \%$ of patients with acute kidney injury requiring renal replacement therapy. No consideration is given for severity of illness scoring, however, and it may be that this reflects a lower threshold for admission of these patients to the ICU. The long-term outcomes of the patients admitted to the ICU were compared with end-stage renal disease patients not requiring ICU care, and unsurprisingly an increase in longterm mortality being associated with heart failure and older age was found. Short-term outcomes (90-day mortality) were associated with the classical cardiovascular risk factors, including diabetes, heart failure and, again, age. This exhibits a similar risk profile to those identified in preoperative studies on end-stage renal patients - in that those patients with limited cardiac reserve fare less well.

Continuing the renal theme, Critical Care Medicine also carries yet another paper examining the role of regional, citrate-based anticoagulation for continuous venovenous haemodialfiltration [9]. In keeping with many studies, the outlook from acute kidney injury in this cohort was bleak with a hospital mortality of $59 \%$ from a cohort of 143 patients. The degree of resulting dialysis dependence was also high, with one in five survivors requiring long-term renal replacement therapy. This is a retrospective noncontrol-based study. Several positive conclusions can, however, be drawn from the study. Firstly, the circuit survival time using this regionalbased system was acceptable, the median time being some 72 hours. Secondly, the delivered dose was in excess of $35 \mathrm{ml} / \mathrm{kg} /$ hour, illustrating that citrate systems do not adversely affect dose delivery. In keeping with other studies, subjects with hepatic impairment coped with the citrate load poorly. Is there any benefit from using citrate anticoagulation? The answer to that question remains to be seen and, given the current studies in place, we shall not have to wait long for the answer.

\section{Competing interests}

The author declares that they have no competing interests.

\section{References}

1. Prescott-Clarke P, Primatest P: Health Survey for England 1996. London: HMSO; 1998.

2. Sakr Y, Madl C, Filipescu D, Moreno R, Groeneveld J, Artigas A, Reinhart K, Vincent JL: Obesity is associated with increased morbidity but not mortality in critical ill patients. Intensive Care Med 2008, 34:1999-2009.

3. Frat J-P, Gissot V, Pagot S, Desachy A, Runge I, Lebert C, Robert R: Impact of obesity in mechanically ventilated patients: a prospective study. Intensive Care Med 2008, 34:1991-1998.

4. Galanos AN, Pieper CF, Kussin PS, Winchell MT, Fulkerson WJ, Harrell FE Jr, Teno JM, Layde P, Connors AF Jr, Phillips RS, Wenger NS: Relationship of body mass index to subsequent mortality among seriously ill hospitalized patients. SUPPORT investigators. The study to understand prognoses and preferences for outcome and risks of treatments. Crit Care Med 1997, 25:1962-1968.

5. Druml W: ICU patients: fatter is better? Intensive Care Med 2008, 34:1961-1963.

6. Ruggenenti P, Schieppatti A, Remuzzi G: Progression, remission, regression of chronic renal diseases. Lancet 2001, 357: 1601-1608.

7. Hutchison CA, Crowe AV, Stevens PE, Harrison DA, Lipkin GW: Case mix, outcome and activity for admissions to intensive care units requiring chronic renal dialysis: a secondary analysis of the ICNRC Case Mix Program Database. Crit Care 2007, 11:R50.

8. Bell M, Granath F, Schön S, Löfberg E, Ekbom A, Martling C-R: End-stage renal disease patients on renal replacement therapy in the intensive care unit: short- and long-term outcome. Crit Care Med 2008, 36:2773-2778.

9. Durão MS, Monte JCM, Batista MC, Oliveira M, lizuka IJ, Santos BF, Pereira VG, Cendoroglo M, Santos OFP: The use of regional citrate anticoagulation for continuous venovenous hemodialfiltration in acute kidney injury. Crit Care Med 2008, 36:30243029 . 